\title{
Active Contour Tracking of Moving Objects Using Edge Flows and Ant Colony Optimization in Video Sequences
}

\author{
Dong-Xian Lai ${ }^{1}$, Yuan-Hsiang Chang ${ }^{2}$, and Zhi-He Zhong ${ }^{1}$ \\ ${ }^{1,2}$ Dept.of Information \& Computer Engineering, Chung Yuan Christian Univ., \\ 200,Chung Pei Rd., Jhingli,32023 Taiwan, R.O.C. \\ \{egria7314, specr0000\} @hotmail.com, \\ changyhaice.cycu.edu.tw
}

\begin{abstract}
Object segmentation and tracking are important techniques in video applications. In this paper, we present a novel system for active contour tracking of moving objects in video sequences. Our method includes preprocessing to identify an initial object contour, and object contour segmentation to refine the contour of the moving object. The edge flows and ant colony optimization are incorporated to improve the efficiency during system convergence. Experimental results demonstrated that our system has achieved the automatic segmentation accuracy of $<1$ pixel on average as compared with manual segmentation results. In summary, our system is particularly useful in segmenting and tracking a moving object without constructing a background model for a video scene. Ultimately, our system could be used in object-based video coding or other analysis such as behavior analysis in video surveillance systems.
\end{abstract}

Keywords: Active contour model, Ant colony optimization, Edge flow, Object tracking.

\section{Introduction}

Object contour segmentation and tracking in video sequences are important parts of video applications and can be used in video compression, motion analysis, pose estimation, or behavior analysis, etc. [1-6] For examples, in object-based video coding, video object contour segmentation can be used to extract foreground objects while eliminating redundant background information for storage, thus achieving a high compression ratio. In video surveillance systems, video object tracking can be used as a preprocessing for pose estimation or behavior analysis in video sequences.

Among the past researches, techniques for the segmentation of object contours are necessary for further analysis [7-10]. Object contour segmentation is generally achieved using techniques of single image segmentation. For examples, Comaniciu and Meer [7] proposed a general nonparametric technique for the analysis of a complex multimodal feature space and the mean-shift clustering was used to extract object contours. Shi and Malik [8] treated object segmentation as a graph partitioning problem and proposed a normalized cut criterion to measure both the total dissimilarity 
between the different groups as well as the total similarity within the group. Xu and Ahuja [9] presented a normalized cuts based algorithm to track object contour, and the algorithm did not require any priori global shape model for tracking objects with deformable shapes and appearances. Kass et al. [10] proposed the use of snakes, which are energy-minimizing splines guided by external constraint forces and influenced by image forces, for the object contour segmentation. In Kass's approach, the choice of initial contour is important. If gradient-based approach is used, the initial contour must be outside and shrunk to the object contour. If region-based approach is used, the initial contour can be inside or outside of the object contour, and expanded or shrunk to the object contour.

In recent years, various approaches to video object tracking have been proposed. A typical approach is to construct a background model such that the model can be used for foreground object extraction in a video scene. For examples, Stauffer and Grimson [11] proposed an approach to model each pixel as a mixture of Gaussians and to evaluate the adaptive Gaussian model which is most likely to be the background. They classified the pixel based on whether the Gaussian distribution is considered as part of the background model and updated the model on-line. Elgammal et al. [12] proposed a nonparametric kernel density estimation to construct statistical representations for scene background and foreground region. Although these approaches can be applied in video object tracking, they are generally limited for scenes with small foreground objects and the background model must be updated constantly.

The objective of this research is aimed to develop a novel system for active contour tracking of moving objects using edge flows and ant colony optimization in video sequences. Our approach is designed in an attempt to segment and track the foreground object in video sequences without constructing and updating a background model. In particular, this system is effective when tracking non-rigid objects (e.g. human) in a scene where the complete background can not be acquired from the whole video sequence.

Section 2 presents the system framework and related methodology. Our system module includes preprocessing and object contour segmentation. Section 3 explains our experimental environment, and results. Section 4 presents conclusion of our approach and discusses potential video applications.

\section{Method}

Our system is designed for the analysis of video sequences that are limited to the following constraints: (1) the surrounding background is static, therefore not a moving background; (2) the camera position is fixed and static (i.e., no panning, tilting, or zooming, etc.); and (3) the boundary between the foreground object and its background is well-defined.

Fig. 1 shows a simplified flow chart in our system framework for active contour tracking of moving objects in video sequences. The system framework can be divided into two parts: (1) preprocessing; and (2) object contour segmentation. The preprocessing includes: region growing, motion image detection, background edge removal, and motion boundary extraction (Fig. 2). The object contour segmentation includes: energy calculation, search space construction, pheromone definition, transition 


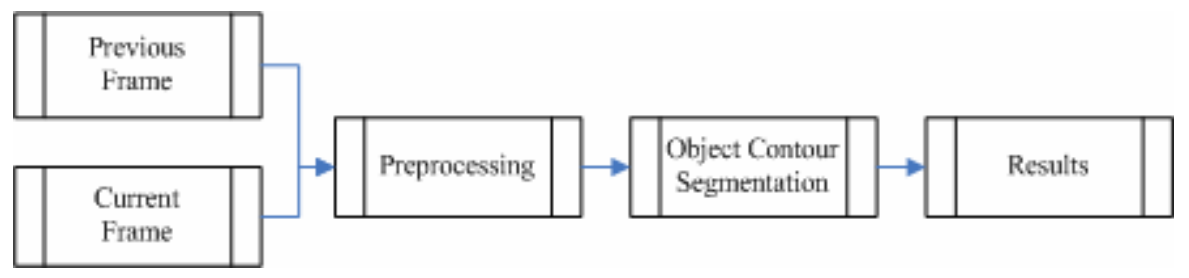

Fig. 1. A simplified flow chart in our system framework for active contour tracking of moving objects in video sequences

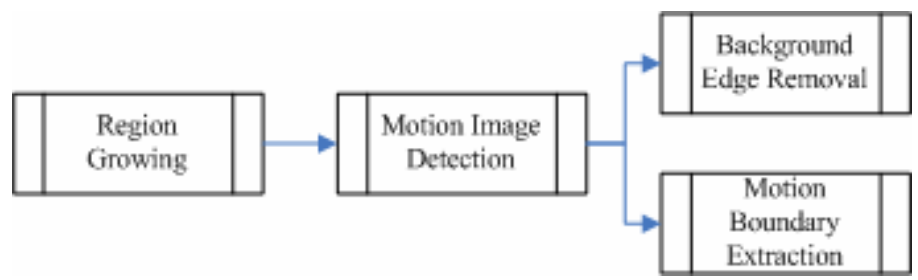

Fig. 2. A simplified flow chart of the preprocessing in our system

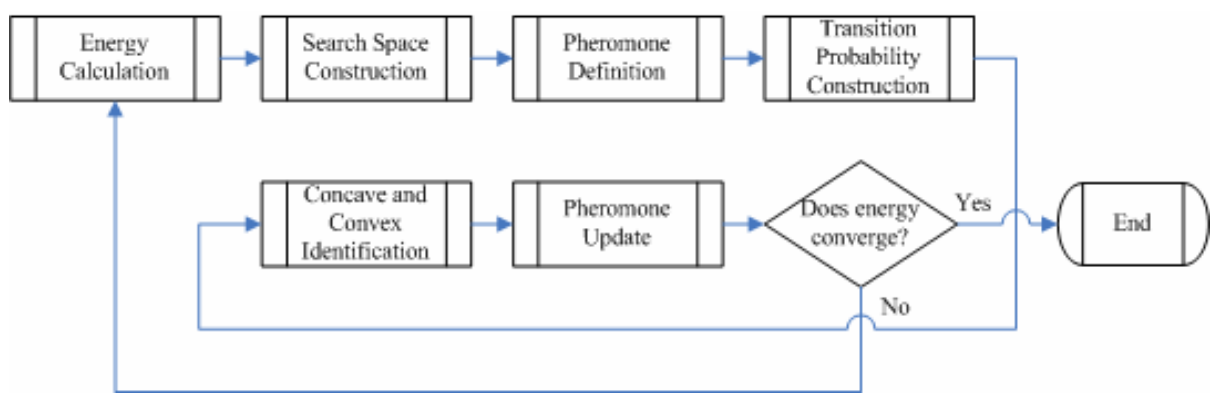

Fig. 3. A simplified flow chart of the object contour segmentation in our system

probability construction, concave and convex identification, pheromone update, and energy convergence criterion (Fig. 3).

\subsection{Preprocessing}

The objective of the preprocessing is to define an initial object contour outside the ideal contour of the moving object in the current frame. The initial object contour is obtained as a combination of the object contour in the previous frame and the region where the object motion occurs in the current frame.

\subsubsection{Region Growing}

In this step, our goal is to find a closed region as the location of the moving object, using the object contour from the previous frame. The processes include: (1) identify the geometric center of the object contour from the previous frame; and (2) use the 


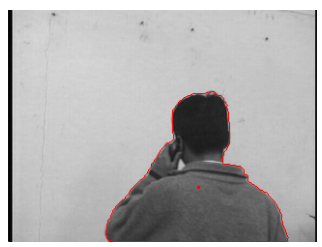

(a)

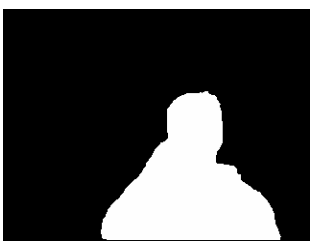

(b)

Fig. 4. An example of the region growing, where (a) contains the contour and the geometric center in the $28^{\text {th }}$ frame from Frank.avi shown in 'red'; and (b) is the resulting image after region growing

geometric center as the seed for region growing to fill inside the contour. An example of the region growing is shown in Fig. 4.

\subsubsection{Motion Image Detection}

In this step, our goal is to detect motion changes near the object contour and construct a motion image using the difference of the previous frame at time $t-1$ and the current frame at time $t$.

Let $I(x, y, t)$ be the gray-level of the image coordinate $(x, y)$ in the frame at time $t, t$ $=1, \ldots, n$, where $n$ is the total number of frames in a video sequence. Then, the difference of frames can be defined by:

$$
d f(x, y, t)= \begin{cases}1 & \text { if }|I(x, y, t)-I(x, y, t-1)| \geq T_{d} \\ 0 & \text { otherwise }\end{cases}
$$

where $T_{d}$ is a threshold. Further, the motion image containing the moving object can be defined by:

$$
M(x, y, t)= \begin{cases}1 & \text { if } d f(x, y, t)=1 \cup \operatorname{gr}(x, y, t-1)=1 \\ 0 & \text { otherwise }\end{cases}
$$

where $\operatorname{gr}(x, y, t-1)$ is the previous frame after region growing ( 1 means foreground, 0 means background). Fig. 5 shows an example of the motion image detection.

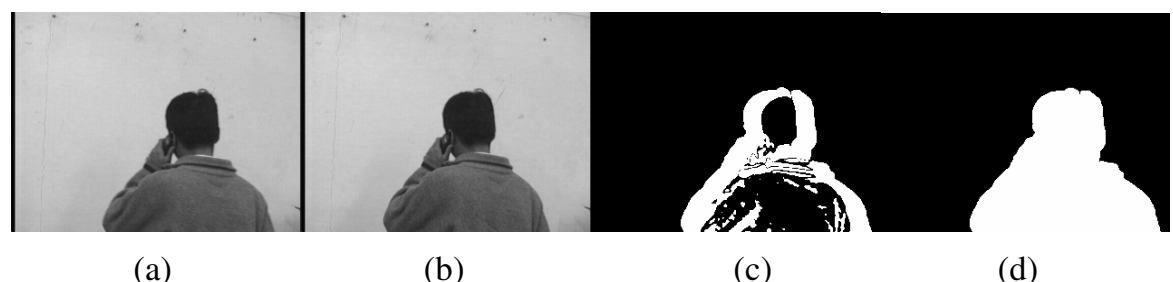

Fig. 5. An example of the motion image detection, where (a) is the original $28^{\text {th }}$ frame from Frank.avi; (b) is the original $29^{\text {th }}$ frame from Frank.avi; (c) is frame difference between 28th and 29th frame after thresholding; and (d) is the resulting image containing the moving object 


\subsubsection{Background Edge Removal}

In this step, our goal is to eliminate the background edges in current frame. Let $I_{\text {edge }}(x$, $y, t)$ be the resulting image of applying the Canny edge detection [13] to the original image $I(x, y, t)$. Then, we remove the background edges and generate the foreground edge image by:

$$
\hat{I}_{\text {edge }}(x, y, t)=\left\{\begin{array}{cl}
I_{\text {edge }}(x, y, t) & \text { if } M(x, y, t)=1 \\
0 & \text { if } M(x, y, t)=0
\end{array}\right.
$$

where $M(x, y, t)$ is used as a mask. This resulting image is later used in the object contour segmentation as the ideal object contour. An example of the background edge removal is shown in Fig .6.

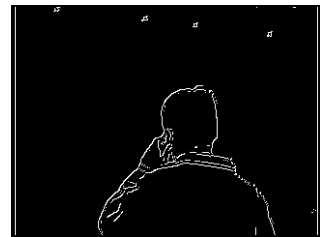

(a)

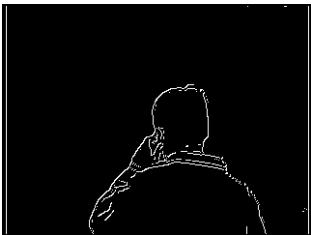

(b)

Fig. 6. An example of the background edge removal, where (a) is the resulting image edge after Canny edge detection in the $29^{\text {th }}$ frame in Frank.avi and (b) is the same image with background edges removed

\subsubsection{Motion Boundary Extraction}

This step attempts to extract a motion boundary of the moving object by:

$$
\hat{I}(x, y, t)=M(x, y, t)-M(x, y, t) \Theta S .
$$

where $S$ is a structuring element and $\Theta$ is the morphological erosion [14]. The resulting image after the motion boundary extraction contains the initial object contour that is slightly larger than the ideal object contour in the current frame. Fig.7 shows an example of the motion boundary extraction.

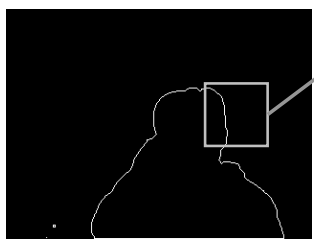

(a)

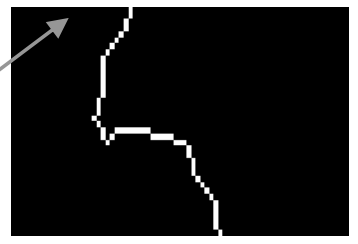

(b)

Fig. 7. An example of motion boundary extraction, where (a) is the motion boundary of $29^{\text {th }}$ frame in Frank.avi; and (b) shows part of the motion boundary in (a) 


\subsection{Object Contour Segmentation}

The purpose of the object contour segmentation is to obtain a well-defined contour for the moving object in each frame. The processes start using the initial object contour in the image $\hat{I}(x, y, t)$ after preprocessing as an active contour model, and iteratively refine the model by the ant colony optimization (ACO) algorithm $[15,16]$. Our approaches incorporate not only the ACO algorithm but also the concept of edge flows [17], in an attempt to improve the efficiency during contour convergence.

\subsubsection{Energy Calculation}

The object contour is iteratively refined by minimizing an energy function. Using the active contour models, the contour curve is defined parametrically as

$$
v(s)=[x(s), y(s)] .
$$

where $x(s)$ and $y(s)$ are $x, y$ coordinates along object contour, and $\mathrm{s} \in[0,1]$. The energy of the object contour can be defined by:

$$
E_{\text {snake }}=\int_{0}^{1} E_{\text {int }}(v(s))+E_{\text {image }}(v(s)) d s .
$$

where $E_{\text {int }}$ represents the internal energy of object contour due to bending, $E_{\text {image }}$ denotes image force. The internal object contour energy can be written as

$$
E_{\text {int }}=w_{\text {elas }}\left|\frac{d v}{d s}\right|^{2}+w_{\text {stiff }}\left|\frac{d^{2} v}{d s^{2}}\right|^{2} .
$$

where $w_{\text {elas }}$ and $w_{\text {stiff }}$ specify the elasticity and stiffness. In addition, the image force $E_{\text {image }}$ can defined by:

$$
E_{\text {image }}=g(x, y)=255-\nabla I_{\text {block }} \text {. }
$$

where $g$ is a function and attracts the object contour with large image gradients, and $\nabla I_{\text {block }}$ is obtained from the Canny gradient image and represent a maximum gradient value in a $3 \times 3$ block at current pixel $(x, y)$. Our goal is to search a contour $\Gamma$ that minimizes the energy function such that:

$$
\Gamma=\arg \min _{s \in(0,1)} E_{\text {snake }}(v(s)) .
$$

\subsubsection{Search Space Construction}

In ACO algorithm, we construct a search space for the ant to find the next possible pixel on the object contour. To improve the efficiency of ACO algorithm, the concept of edge flows is incorporated in the selection of the search space and the processes can be described as follows:

Step 1: Randomly place an ant on the current object contour.

Step 2: Acquire the next contour pixel and compute its edge flow direction. 
Step 3: Select a number of candidate pixels along the edge flow direction as the search space for the ant to explore.

Step 4: Check if the candidate pixels are close to the ideal object contour defined in $\hat{I}_{\text {edge }}(x, y, t)$.

Step 5: If yes, the algorithm terminates. If not, search for all eight directions and re-select the candidate pixels along the direction closest to the ideal object contour.

The use of edge flow is originally proposed by Ma and Manjunath [17]. The direction of edge flow is computed by:

$$
\theta(x, y, t)=\arg \max _{\theta}\left\{\sum_{\theta \leq \theta^{<}<\theta+\pi} P\left(x, y, t, \theta^{\prime}\right)\right\} .
$$

where $P\left(x, y, t, \theta^{\prime}\right)$ represents the probability of finding edge along orientation $\theta^{\prime}$ at location $(x, y)$, and is defined by:

$$
P(x, y, t, \theta)=\frac{\operatorname{Error}(x, y, t, \theta)}{\operatorname{Error}(x, y, t, \theta)+\operatorname{Error}(x, y, t, \theta+\pi)} .
$$

where $\operatorname{Error}(x, y, t, \theta)$ represents the prediction error along orientation $\theta$, and is defined by:

$$
\operatorname{Error}(x, y, t, \theta)=\left|I_{\sigma}(x+d \cos \theta, y+d \sin \theta, t)-I_{\sigma}(x, y, t)\right| .
$$

where $I_{\sigma}(x, y, t)$ represents the frame at time $t$ after Gaussian smoothing with $\sigma$ and $d$ is a distance of prediction and proportional of $\sigma$, in the system we assign $d=4 \sigma$.

\subsubsection{Pheromone Definition}

In ACO algorithm, pheromone is defined to help ants communicate with each other, and the concentration of pheromone can influence ants to explore in the next iteration. The initial concentration of pheromone is defined by:

$$
\tau_{i j}=\frac{1}{E_{\text {snake }}\left(\Gamma_{k}\right)} .
$$

where $i$ is the pixel in the image $I(x, y, t)$ and $j$ is its neighboring pixel, and $\Gamma_{k}$ represents object contour at the $k$-th iteration.

\subsubsection{Transition Probability Construction}

For the ant to explore the next pixel on the object contour, a transition probability is assigned to each candidate pixel in the search space as defined by:

$$
P_{i j}=\left\{\begin{array}{cl}
\frac{\tau_{i j}^{\alpha} \cdot \eta_{i j}^{\beta}}{\sum_{l \in \text { candidate pixels }}^{\alpha} \tau_{i l}^{\alpha} \cdot \eta_{i}^{\beta}} & \text { if } j \in \text { candidate pixels } \\
0 & \text { otherwise }
\end{array} .\right.
$$


where $i$ represents current pixel on object contour, and $j$ represents the next pixel to explore in the search space. $\tau_{i j}$ represents the concentration of pheromone between pixels $i$ and $j . \eta_{i j}$ represents the visibility and equal to reciprocal of distance between pixels $i$ and $j$. $\alpha$ represents the weight of pheromone concentration, and $\beta$ represents the weight of visibility.

\subsubsection{Concave and Convex Processing}

To insure that the ant will explore in the direction more closely to the ideal object contour, we incorporate a criterion for three consecutive pixels $\mathrm{v}_{\mathrm{i}-1}, \mathrm{v}_{\mathrm{i}}$, and $\mathrm{v}_{\mathrm{i}+1}$ in two difference situations, namely the concave and the convex cases. Fig. 8 shows the two different cases which can be described as follows:

(a) Concave case: In this case, we check if the pixels on the straight-line from $v_{i}$ to $\mathrm{v}_{\mathrm{i}+1}$ are associated with opposite edge flow directions between two neighboring pixels. If yes, each pixel on the straight-line finds the closest point on the ideal contour and choose the closest one as the next pixel to explore; if not, the straight-line remains unchanged.

(b) Convex case: In this case, we simply check whether $v_{i+1}$ is close to the ideal object contour. If yes, do nothing; if not, find the pixels close to the ideal object contour along eight possible directions and choose the closest one as the next pixel to explore.

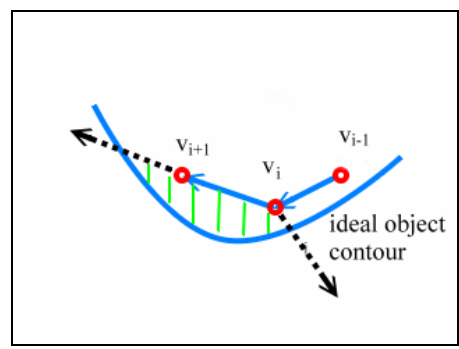

(a)

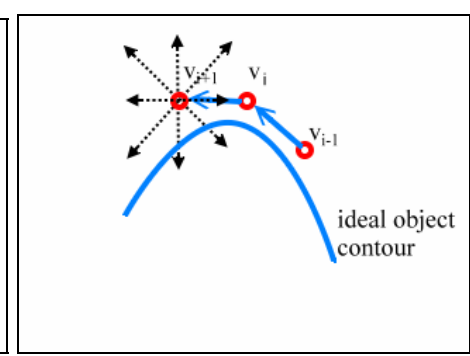

(b)

Fig. 8. (a) concave case; (b) convex case. The ideal object contour is obtained by edge detection, and $\mathrm{v}_{\mathrm{i}-1}, \mathrm{v}_{\mathrm{i}}$, and $\mathrm{v}_{\mathrm{i}+1}$ are represent three consecutive pixels in the current object contour.

\subsubsection{Pheromone Update}

In this step, we update the concentration of pheromone on the object contour to make the best contour so far more probable to be selected in the next iteration. In our approach, the new object contour with smaller energy is used to replace old contour with larger energy at each iteration. In addition, pheromone on the contour is evaporated and the concentration is decreased. The algorithm can be simply described by: 


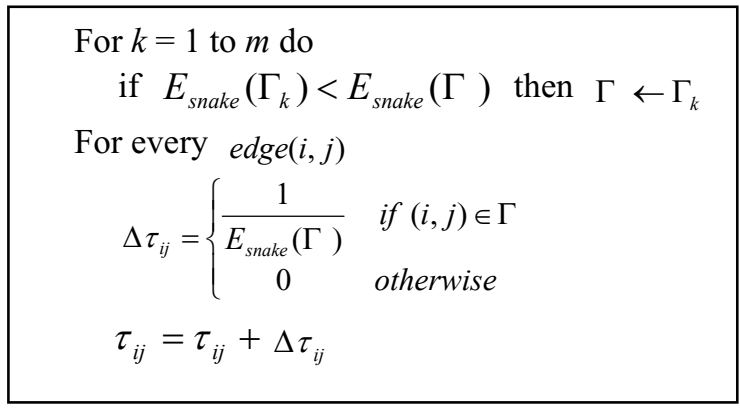

where $k$ represents the iteration number, $\Gamma$ represents the best contour so far.

\subsubsection{Energy Convergence}

At each iteration, our system check if the following criterion satisfies:

$$
\frac{\left|E_{\text {snake }}\left(\Gamma_{k}\right)-E_{\text {snake }}(\Gamma)\right|}{E_{\text {snake }}(\Gamma)}<\varepsilon .
$$

where $\varepsilon$ is the convergence threshold. If yes, the system outputs the object contour segmentation result. Otherwise, the system repeats the processes again.

Fig. 9 shows an example of the object contour segmentation for the $29^{\text {th }}$ frame of Frank.avi. The human object is moving from right to left.

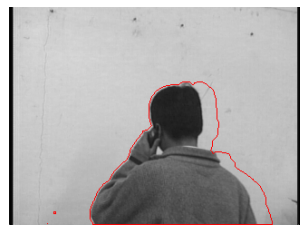

(a)

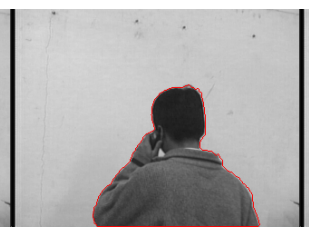

(b)

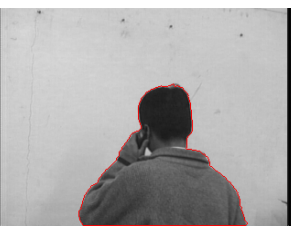

(c)

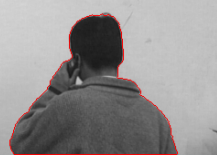

(d)

Fig. 9. An example of the object contour segmentation for the $29^{\text {th }}$ frame of Frank.avi, after preprocessing; where (a) is initial object contour after preprocessing; (b) is the object contour after the $1^{\text {st }}$ iteration; (c) is the object contour at the $2^{\text {nd }}$ iteration; and (d) is the resulting object contour after convergence

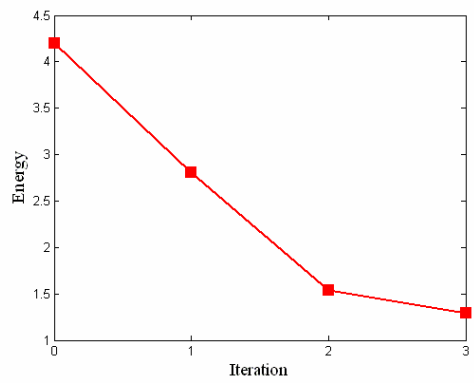

Fig. 10. The corresponding energy as computed during system convergence for the object contour segmentation shown in Fig. 9 


\subsection{System Evaluation}

To evaluate our system, we quantitatively measure the similarity between two segmentation results, i.e., manually and automatically segmented results, using the root mean square (RMS) error by:

$$
e_{R M S}=\sqrt{\frac{1}{N} \sum_{i=1}^{N} d\left(\hat{P}_{i}, P_{i}\right)^{2}}
$$

where $N$ represents the number of pixels on the manually segmented contour, $\hat{P}_{i}$ represents the $i$-th pixel on the manually segmented contour, and $P_{i}$ represents the pixel on the automatically segmented contour that is the closest pixel to the $i$-th pixel in manually segmented contour. $d\left(\hat{P}_{i}, P_{i}\right)$ means the Euclidean distance between the two pixels $\hat{P}_{i}$ and $P_{i}$. A small RMS error means the two segmented contour are closely matched.

\section{Results}

The video sequences used for our system evaluation are with the two kinds of resolutions: $320 \times 240$ pixels or $176 \times 144$ pixels in each video frame. All the original video sequences contain 24-bit color video frames, that have been previously processed to gray-level images prior to the object contour segmentation and tracking. Table 1 summaries the system parameters used in our system for active contour tracking of moving objects.

Table 1. System parameters used in the active contour tracking of moving object

\begin{tabular}{|c|c|c|}
\hline \multicolumn{2}{|c|}{ Preprocessing } & \multicolumn{2}{c|}{ Object Contour Segmentation } \\
\hline$T_{d}$ & $w_{\text {elas }}$ & 1.0 \\
\hline \multirow{3}{*}{10} & $w_{\text {stiff }}$ & 1.0 \\
\cline { 2 - 3 } & $\sigma$ & 1.0 \\
\cline { 2 - 3 } & $\alpha$ & 1 \\
\cline { 2 - 3 } & $\beta$ & 1 \\
\cline { 2 - 3 } & $\mathcal{E}$ & 0.2 \\
\hline
\end{tabular}

Experimental results of the object contour segmentation are shown in Fig. 11 (Frank.avi) and Fig. 12 (Akiyo.avi), respectively. The segmented object contour is highlighted in 'red'. To evaluate our system, the object contour as automatically segmented is compared with the object contour as extracted manually. Because manual segmentation is too tedious, we only compute the RMS error in selected frames. The contour of the moving object is manually extracted using commercially available software (i.e., PhotoImpact) by an observer. Table 2 and 3 show the RMS errors of selected frames between the manual and automatic segmentation results. 


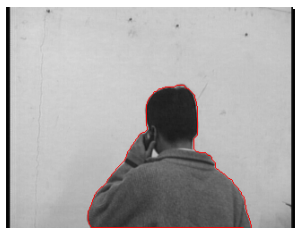

(a)

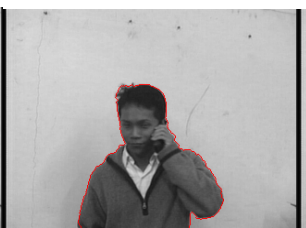

(b)

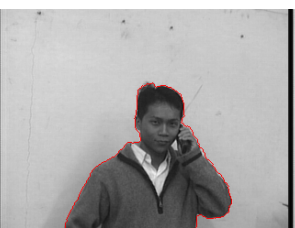

(c)

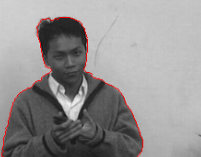

(d)

Fig. 11. Segmentation results of object contour in the (a) $29^{\text {th }}$, (b) $39^{\text {th }}$, (c) $49^{\text {th }}$, and (d) $59^{\text {th }}$ frame from Frank.avi, where the segmented object contour is highlighted in 'red'

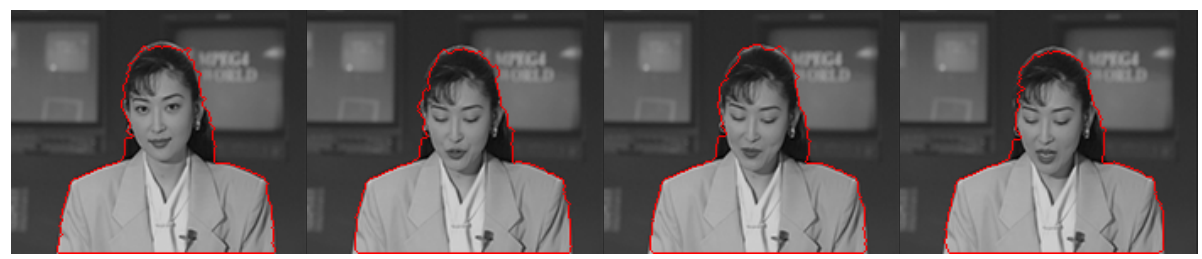

(a)

(b)

(c)

(d)

Fig. 12. Segmentation results of object contour in the (a) $2^{\text {nd }}$, (b) $42^{\text {nd }}$, (c) $82^{\text {nd }}$ and (d) $122^{\text {nd }}$ frame from Akiyo.avi, where the segmented object contour is highlighted in 'red'

Table 2. The RMS error of selected frames between the manual and automatic segmentation results in Frank.avi. The mean and standard deviation of the RMS error are also shown (Unit: pixels).

\begin{tabular}{|c|c|c|c|c|c|c|}
\hline & \multicolumn{4}{|c|}{ Frame No. of Frank.avi } & Mean & $\begin{array}{c}\text { Standard } \\
\text { Deviation }\end{array}$ \\
\hline & $29^{\text {th }}$ & $39^{\text {th }}$ & $49^{\text {th }}$ & $59^{\text {th }}$ & & \\
\hline$e_{R M S}$ & 1.12 & 0.96 & 0.95 & 0.78 & 0.95 & 0.12 \\
\hline
\end{tabular}

Table 3. The RMS error of selected frames between the manual and automatic segmentation results in Akiyo.avi

\begin{tabular}{|c|c|c|c|c|c|c|}
\hline & \multicolumn{4}{|c|}{ Frame No. of Akiyo.avi } & Mean & $\begin{array}{c}\text { Standard } \\
\text { Deviation }\end{array}$ \\
\hline & $2^{\text {th }}$ & $42^{\text {th }}$ & $82^{\text {th }}$ & $122^{\text {th }}$ & & \\
\hline$e_{R M S}$ & 0.76 & 1.06 & 0.91 & 0.96 & 0.92 & 0.22 \\
\hline
\end{tabular}

\section{Conclusion}

In this paper, we present a novel system for the active contour tracking of moving objects using edge flows and ant colony optimization in video sequences. Our system incorporates the use of edge flows in the ACO algorithm such that the efficiency could be improved. The method is particularly useful in segmenting and tracking a moving object (e.g., human) without constructing a background model for a video 
scene. Our experimental results demonstrated that our system has achieved the automatic segmentation accuracy of $<1$ pixel on average as compared with the manual segmentation results. Ultimately, our system could be used to identify foreground objects from their background, leading to an effective object-based video coding (e.g., H.264/AVC). In addition, if the contour of the moving object can be extracted, further analysis (e.g., behavior analysis, video content retrieval, or object tracking and recognition) can therefore be performed based on the moving object in a video surveillance system. However, our system was designed assuming the camera is static, other techniques (e.g., video stabilization [18]) may be required before defining the object contour in real video scenes (e.g., video vibration induced by unwanted camera motion).

\section{References}

1. Xiang, T., Gong, S.: Video behavior profiling for anomaly detection. IEEE Trans. Pattern Anal. Mach. Intell. 30, 893-908 (2008)

2. Gupta, A., Mittal, A., Davis, L.S.: Constraint integration for efficient multiview pose estimation with self-occlusions. IEEE Trans. Pattern Anal. Mach. Intell. 30, 493-506 (2008)

3. Sundaramoorthi, G., Yezzi, A., Mennucci, A.C.: Coarse-to-fine segmentation and tracking using sobolev active contours. IEEE Trans. Pattern Anal. Mach. Intell. 30, 851-864 (2008)

4. Zhao, T., Nevatia, R., Wu, B.: Segmentation and tracking of multiple humans in crowded environments. IEEE Trans. Pattern Anal. Mach. Intell. 30, 1198-1211 (2008)

5. Han, B., Comaniciu, D., Zhu, Y., Davis, L.S.: Sequential kernel density approximation and its application to real-time visual tracking. IEEE Trans. Pattern Anal. Mach. Intell. 30, 1186-1197 (2008)

6. Briassouli, A., Ahuja, N.: Extraction and Analysis of multiple periodic motions in video sequence. IEEE Trans. Pattern Anal. Mach. Intell. 29, 1244-1261 (2007)

7. Comaniciu, D., Meer, P.: Mean shift: A robust approach toward feature space analysis. IEEE Trans. Pattern Anal. Mach. Intell. 24, 603-619 (2002)

8. Shi, J., Malik, J.: Normalized cuts and image segmentation. IEEE Trans. Pattern Anal. Mach. Intell. 22, 888-905 (2000)

9. Xu, N., Ahuja, N.: Object contour tracking using graph cuts based active contours. In: IEEE Proceedings International Conference on Image Processing, vol. 3, pp. III-277-III280 (2002)

10. Kass, M., Witkin, A., Terzopoulos, D.: Snakes: Active contour models. International Journal of Computer Vision 1, 321-331 (1988)

11. Stauffer, C., Grimson, W.: Adaptive background mixture models for real-time tracking. In: IEEE Computer Society Conference on Computer Vision and Pattern Recognition, vol. 2, p. 252 (1999)

12. Elgammal, A., Duraiswami, R., Hardwood, D., Davis, L.S.: Background and foreground modeling using nonparametric kernel density estimation for visual surveillance. IEEE Proceeding 90(7), 1151-1163 (2002)

13. Canny edge detection tutorial (2008), http://www.pages.drexel.edu/ weg22/can_tut.html

14. Gonzalez, R.C., Wood, R.E.: Digital Image Processing, 2nd edn. Prentice Hall, New Jersey (2002)

15. Dorigo, M., Maniezzo, V., Colorni, A.: The ant system: Optimazation by a colony of cooperating agents. IEEE Trans. Systems, Man, and Cybernetics 26, 29-41 (1996) 
16. Wang, X.N., Feng, Y.J., Feng, Z.R.: Ant colony optimization for image segmentation. In: IEEE Proceeding International conference on Machine Learning and Cybernetics, vol. 9, pp. 5355-5360 (2005)

17. Ma, W.Y., Manjunath, B.S.: Edge flow: A framework of boundary detection and image segmentation. In: IEEE Proceedings. Computer Society Conference on Computer Vision and Pattern Recognition, pp. 744-749 (1997)

18. Litvin, A., Konrad, J., Karl, W.C.: Probabilistic video stabilization using kalman filtering and mosaicking. In: Proceedings of SPIE-IS\&T Electronic Imaging, SPIE, vol. 5002, pp. 663-674 (2003) 